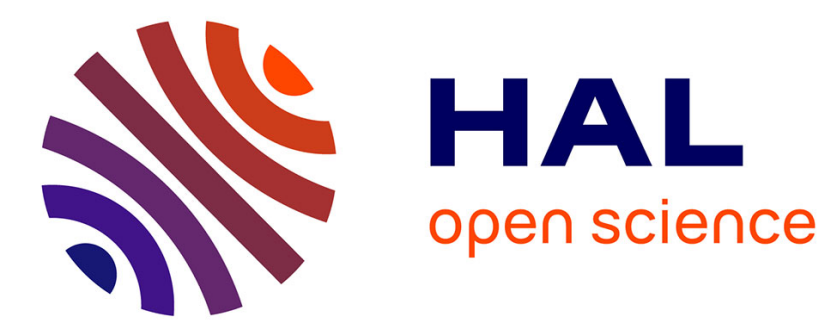

\title{
Load carrying capacity of systems within a global safety perspective. Part I. Robustness of stable equilibria under imperfections
}

Stefano Lenci, Giuseppe Rega

\section{- To cite this version:}

Stefano Lenci, Giuseppe Rega. Load carrying capacity of systems within a global safety perspective. Part I. Robustness of stable equilibria under imperfections. International Journal of Non-Linear Mechanics, 2011, 46 (9), pp.1232. 10.1016/j.ijnonlinmec.2011.05.020 . hal-00784916

\author{
HAL Id: hal-00784916 \\ https://hal.science/hal-00784916
}

Submitted on 5 Feb 2013

HAL is a multi-disciplinary open access archive for the deposit and dissemination of scientific research documents, whether they are published or not. The documents may come from teaching and research institutions in France or abroad, or from public or private research centers.
L'archive ouverte pluridisciplinaire HAL, est destinée au dépôt et à la diffusion de documents scientifiques de niveau recherche, publiés ou non, émanant des établissements d'enseignement et de recherche français ou étrangers, des laboratoires publics ou privés. 


\section{Author's Accepted Manuscript}

Load carrying capacity of systems within a global safety perspective. Part I. Robustness of stable equilibria under imperfections

Stefano Lenci, Giuseppe Rega

PII: $\quad$ S0020-7462(11)00149-1

DOI: doi:10.1016/j.ijnonlinmec.2011.05.020

Reference: $\quad$ NLM 1891

To appear in: International Journal of Non-

Linear Mechanics

Received date: 20 February 2011

Revised date: 26 April 2011

Accepted date: 7 May 2011

Cite this article as: Stefano Lenci and Giuseppe Rega, Load carrying capacity of systems within a global safety perspective. Part I. Robustness of stable equilibria under imperfections, International Journal of Non-Linear Mechanics, doi:10.1016/j.ijnonlinmec.2011.05.020

This is a PDF file of an unedited manuscript that has been accepted for publication. As a service to our customers we are providing this early version of the manuscript. The manuscript will undergo copyediting, typesetting, and review of the resulting galley proof before it is published in its final citable form. Please note that during the production process errors may be discovered which could affect the content, and all legal disclaimers that apply to the journal pertain. 


\title{
LOAD CARRYING CAPACITY OF SYSTEMS WITHIN A GLOBAL SAFETY PERSPECTIVE. PART I. ROBUSTNESS OF STABLE EQUILIBRIA UNDER IMPERFECTIONS
}

\author{
Stefano Lenci \\ Department of Architecture, Buildings and Structures \\ Polytechnic University of Marche, Ancona, Italy, lenci@univpm.it \\ Giuseppe Rega \\ Department of Structural and Geotechnical Engineering \\ Sapienza University of Rome, Rome, Italy, Giuseppe.Rega@uniromal.it
}

\begin{abstract}
The problem of the practical stability of structures is addressed in a modern way by considering the effects of both static and dynamic perturbations. The major historical contributions, due to Euler, Koiter and Thompson, are reviewed and illustrated by an archetypal model permitting to highlight the main mechanical and dynamical points. It is found that a global approach is necessary for a reliable safety estimation, especially in the neighborhood of (static) critical loads. Considering that the admissible load threshold has to account for robustness to finite perturbations, the Koiter critical load must be lowered, obtaining the so called Thompson critical load. It is shown how these two thresholds share some properties (e.g. both depend in a sensitive way on imperfections, which must be known for practical calculations), while having a deep different meaning: the former is related to static imperfections, and requires only a local analysis, while the latter is related to dynamical imperfections, and requires a global analysis. It is shown that $p_{\text {Euler }}^{\text {crit }} \geq p_{\text {Koiter }}^{\text {crit }} \geq p_{\text {Thompson }}^{\text {crit }}$, i.e., that the advancement of knowledge leads to a lower estimation of the actual critical load.
\end{abstract}

Keywords: Practical stability, static imperfections, global safety, robustness of equilibria, Euler, Koiter, Thompson.

\section{INTRODUCTION}

Determining the load carrying capacity of a compressed structural element (columns, frames, shells, etc.) is an old problem which dates back at least to Euler [1]. It was a long history of successes and defeats of scientists which, in the authors' opinion, still extends over the present time.

The first fundamental contribution was due to Euler [1] which determined the famous Euler buckling load of a column. The loss of load carrying capacity was identified as the bifurcation point from an equilibrium path in the parameters space - talking, of course, in modern language. Although the concept of stability was formulated in a rigorous way only much later, with the major contribution, among others, of Lyapunov [2], it is felt that the main idea of loss of stability was already lurking in Euler's background. We quote [3] for an interesting historical note on the development of the stability concepts. 
The second outstanding contribution was due to Koiter [4], who realized that imperfections are crucial in lowering the critical load. This basic idea, and successive developments, were so important that investigations in this direction continue up to date [5]. In practice, due to imperfections, the branching point becomes a snap point, which occurs at a lower load threshold. Later on, the bifurcation theory has provided a mathematical background to this engineering intuition, by rigorously showing that transcritical and pitchfork bifurcations (responsible for branching) are structurally unstable events, which become saddle-node bifurcations (responsible for snap) after system perturbations (imperfections in mechanical language). Structural stability concepts are part of the more general catastrophe theory $[6,7]$. In this context, though the general theorems are very complex and abstract, the basic idea is simply that of studying perturbations of the system with respect to parameters and not to initial conditions, as in classical local stability.

Although at Koiter's time it was clear that stability is a dynamical concept [8], the major initial contributions were concerned with a "static" stability approach $[9,10]$. When 'flutter' or 'galloping' came to the attention of researchers, dynamics entered the question of loss of stability (see [11] for a theoretical approach and [12] for a practical approach). In the bifurcation theory language, the Hopf bifurcation was 'discovered' to exist in practice, and this agrees with the fact that it is a structurally stable event which can be actually seen to occur as, e.g., in the dramatic failure of the Tacoma Bridge or in other aero-elastically induced collapses of structures.

This basic, and necessarily over-summarized, evolution of knowledge is clearly understood (see, e.g., [13]), and it is apparent that only local bifurcational events were concerned, indeed.

It was Thompson that, around the 90's [14-16], discovered that (local) stability is not enough, and that the relevant results do not actually guarantee the load carrying capacity of systems. By considering a global approach, and by truly considering the dynamical behavior (even in the absence of an external excitation), Thompson introduced the notion of dynamical integrity, which in the authors' opinion is fundamental for properly pursuing the safety of structures in an evolutionary context, although to date it is not yet a commonly addressed concept.

The basic idea is that perturbations can be also of dynamical nature, and not only of static nature as in Koiter's vision. This means that not only the system parameters and equations can be modified by the imperfections, but that also the initial conditions of the system may change (in this respect somehow embedding the classical stability concepts into a structural stability perspective). From this observation, Thompson realized two main points.

(i) If the safe basin of a given, desirable, solution is not 'large' enough, even if the solution is stable there will be no hope to observe it in real world applications - maybe only in extremely accurate experiments. This was his first fundamental understanding, which can be reformulated by saying that classical local stability refers to infinitesimal changes in initial conditions, while global safety 
refers to finite changes in initial conditions. Thus, local stability must be complemented by robustness towards variations of the initial conditions.

(ii) A second fundamental requirement for actual global safety is that the safe basin, or the basin of attraction under an actual dynamic excitation, must be 'uncorrupted' (or dynamically integer, i.e., non-fractal) in order to achieve a reliable load carrying capacity.

Since the safe basin shrinks to the equilibrium solution when a saddle-node bifurcation is approached, the saddle-node (Koiter) threshold is expected to overestimate the actual critical load in the presence of (even transient) dynamical perturbations.

Within the above mentioned overall framework, this paper, which is the Part I of this work, aims at addressing the sole first point of the two Thompson's achievements, by embedding it into an historical perspective and showing how, as Koiter lowered the buckling load prediction of Euler, Thompson lowers the buckling load prediction of Koiter. Indeed, the analysis of the robustness of stable equilibria can be investigated by considering the global dynamics of the system even in the absence of dynamical excitation, which therefore is not considered in this Part I. A goal consists of highlighting how the problem of the load carrying capacity of structures can be actually understood in all of its implications, at least from a theoretical point of view, only if finite dynamical perturbations are taken into account. This is addressed herein by considering an archetypal single degree-of-freedom model which exhibits a transcritical static bifurcation in perfect (Euler) conditions, a fold static bifurcation in imperfect (Koiter) conditions, and competing families of inwell and cross-well oscillations in dynamic (Thompson) conditions. It is shown how the consideration of finite dynamical perturbations, in addition to static ones, notably affects the system safety, giving rise to a Thompson critical load which is always considerably lower than the Koiter one (and, of course, than the Euler one).

Dynamic excitations, on the other hand, entail strong modifications to the topology of the basins, which are related to their dynamical integrity and which further reduce the safety of the system. This issue, which constitutes the second point of the Thompson achievements, entails properly addressing the dynamical integrity concept in all of its implications, which are indeed quite delicate because of the strange dynamical phenomena (e.g., fractals) which may occur. This will be considered in the companion paper [17], by also addressing the so-called integrity (or basin erosion) profiles, which are very useful instruments to achieve the reliability threshold, as well as the most important practical information ensuing from the analysis of system dynamics. Part II [17] complements this one and concludes the analysis of the global safety.

The paper is organized as follows. In Sect. 2 the archetypal problem is illustrated together with the governing equations and relevant mechanical properties. In Sect. 3 the Euler critical load is determined in the perfect case, while in Sect. 4 the imperfections are added and the Koiter critical 
load computed. The Thompson analysis, taking care of possible (dynamic) finite perturbations of initial conditions, is performed in Sect. 5. In this framework, an asymptotic analysis highlighting the sensitivity of the problem to both static and dynamic perturbations is developed in Sect. 5.1. The paper ends with some conclusions (Sect. 6).

\section{THE ARCHETYPAL MODEL}

Let us consider the single degree-of-freedom mechanical system described in Fig. 1, where $I$ is the moment of inertia with respect to the hinge $B, K$ the stiffness of the linear spring, $H$ the length of the rigid beam, $L$ the horizontal distance between the hinge $B$ and the extreme point of the spring $A$, and $\beta$ is the angle of rotation which describes the system configuration.

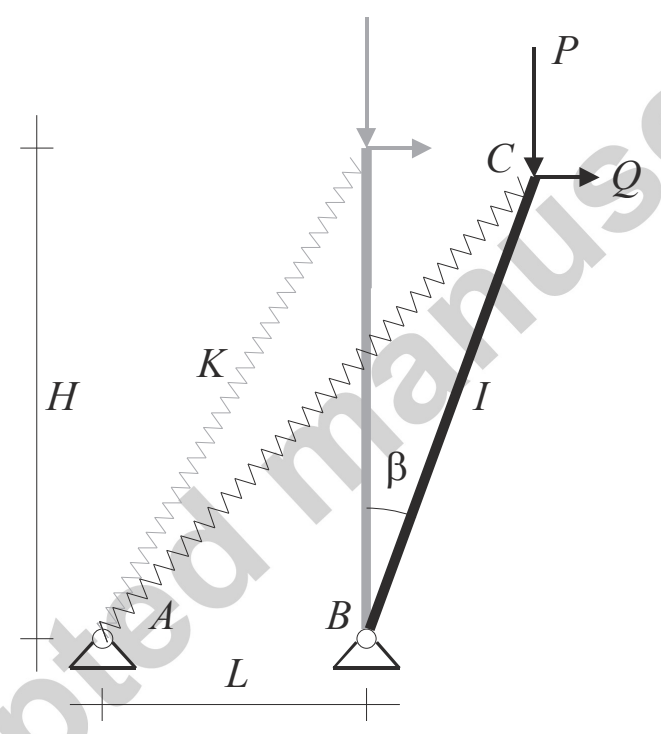

Fig. 1. The mechanical model.

By considering the dimensionless time $t=\hat{t} \sqrt{K L H / I}$, vertical $p=P / K L$ and horizontal $q=Q / K L$ loads, and by introducing the dimensionless parameter $\alpha=2 L H /\left(L^{2}+H^{2}\right)$ (which satisfies $0<\alpha \leq 1$ and $\alpha=1$ if and only if $L=H$ ) the dimensionless kinetic energy, elastic potential energy and load potential work are given by

$$
\begin{gathered}
T=\frac{\hat{T}}{K L H}=\frac{1}{2} \dot{\beta}^{2}, \\
U=\frac{\hat{U}}{K L H}=\frac{1}{\alpha}[1-\sqrt{1+\alpha \sin (\beta)}]^{2}, \\
W=\frac{\hat{W}}{K L H}=p[1-\cos (\beta)]+q \sin (\beta),
\end{gathered}
$$


where the dot means derivative with respect to the dimensionless time $t$. The stationariety of the action integral

$$
l=\int_{t_{1}}^{t_{2}}[T(t)-U(t)+W(t)] d t
$$

with respect to $\beta(t)$ yields the equation of motion

$$
\ddot{\beta}-p \sin (\beta)+\left[1-\frac{1}{\sqrt{1+\alpha \sin (\beta)}}-q\right] \cos (\beta)=0 .
$$

In general both $p$ and $q$ can be time dependent. However, when they are time independent the system is conservative and the energy $E=T+U \quad W$,

$$
E=\frac{\dot{\beta}^{2}}{2}+\frac{[1-\sqrt{1+\alpha \sin (\beta)}]^{2}}{\alpha}-p[1-\cos (\beta)]-q \sin (\beta),
$$

is constant along trajectories. The potential $V=U \quad W$,

$$
V=\frac{[1-\sqrt{1+\alpha \sin (\beta)}]^{2}}{\alpha}-p[1-\cos (\beta)]-q \sin (\beta),
$$

is drawn in Fig. 2 for various values of the parameters. The zoom of Fig. $2 b$ shows that the rest position is not an equilibrium, due to the imperfection $q \neq 0$, and how the non null equilibrium position loses stability for increasing $p$.

The equilibrium points are given by

$$
p \sin (\beta)=\left[1-\frac{1}{\sqrt{1+\alpha \sin (\beta)}}-q\right] \cos (\beta)
$$

and the associated bifurcation diagrams for perfect $(q=0)$ and imperfect cases ( $q \neq 0$ but constant) are reported in Figs. 3 and 4, respectively. A joint zoom, permitting to visualize the effects of the imperfections, is reported in Fig. 5. 


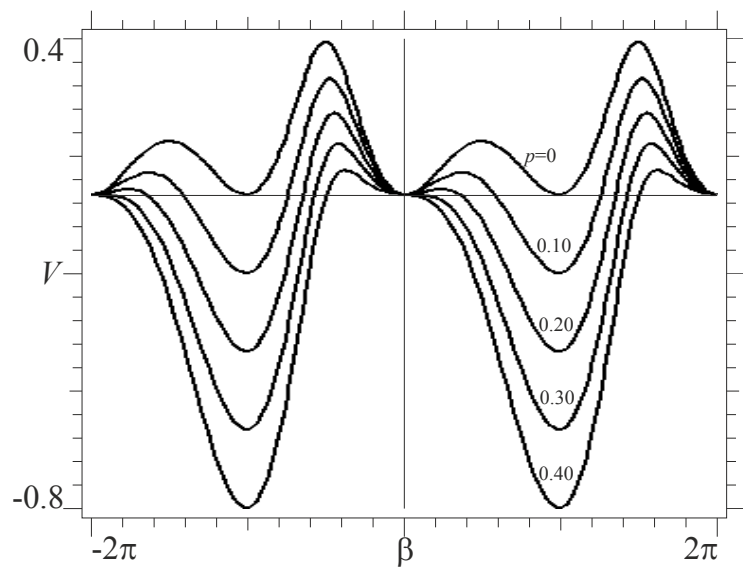

(a)

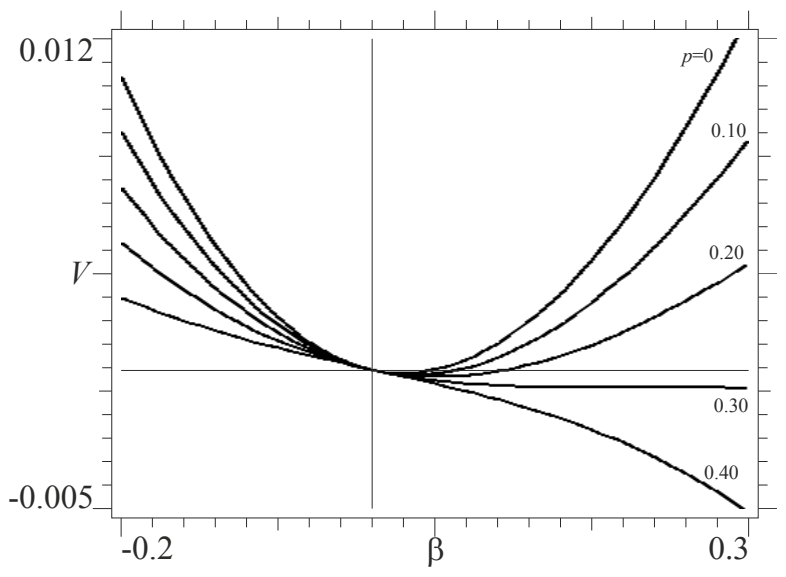

(b)

Fig. 2. The potential $V(\beta)$ for $q=0.01, \alpha=0.8$ and for different values of $p$. (a) Large view, which in particular underlines the $2 \pi$ periodicity with respect to $\beta$, and (b) zoom around $\beta=0$.

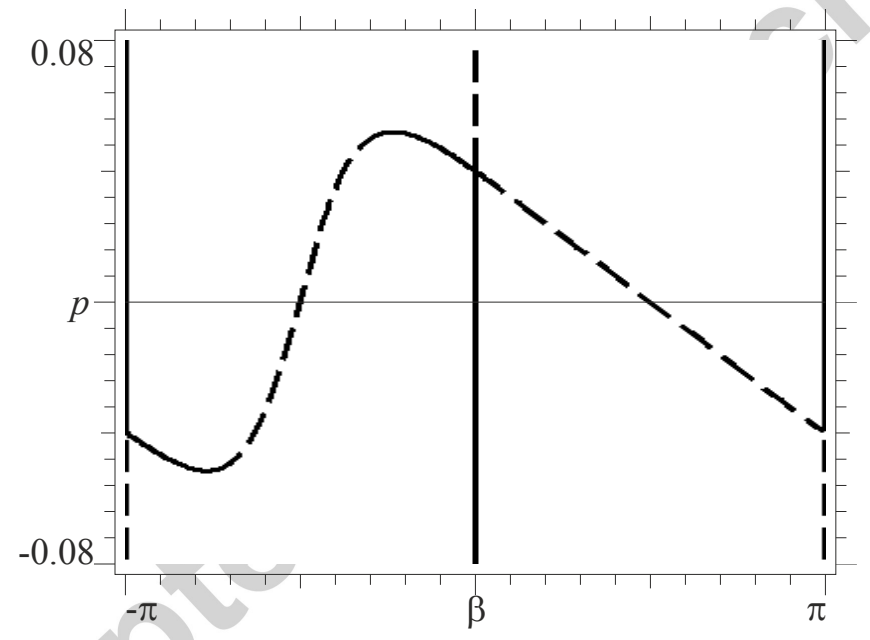

Fig. 3. The complete bifurcation diagram in the perfect case $q=0 . \alpha=0.8$, continuous $=$ stable, dashed $=$ unstable.

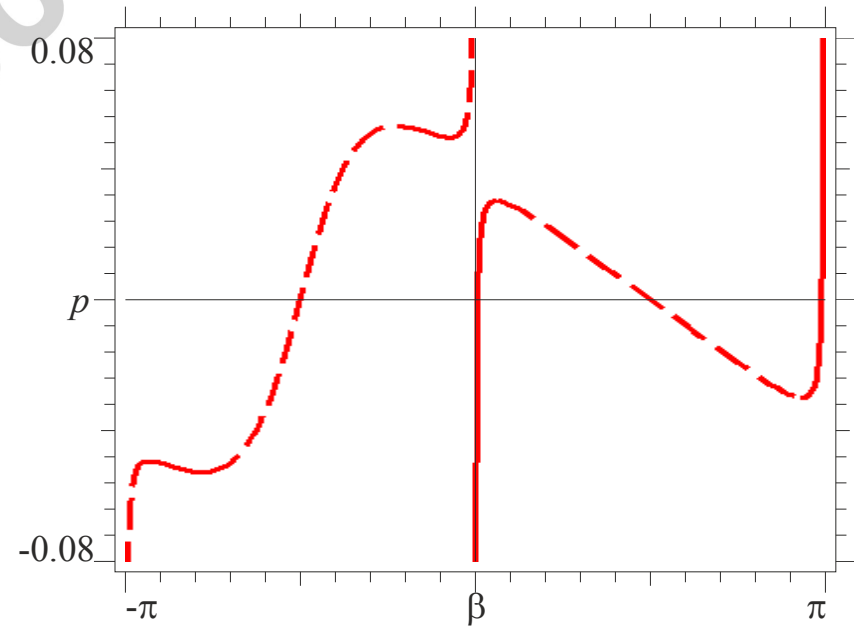

Fig. 4. (color online) The complete bifurcation diagram in the imperfect case $q=0.01 . \alpha=0.8$, continuous $=$ stable, dashed $=$ unstable. 


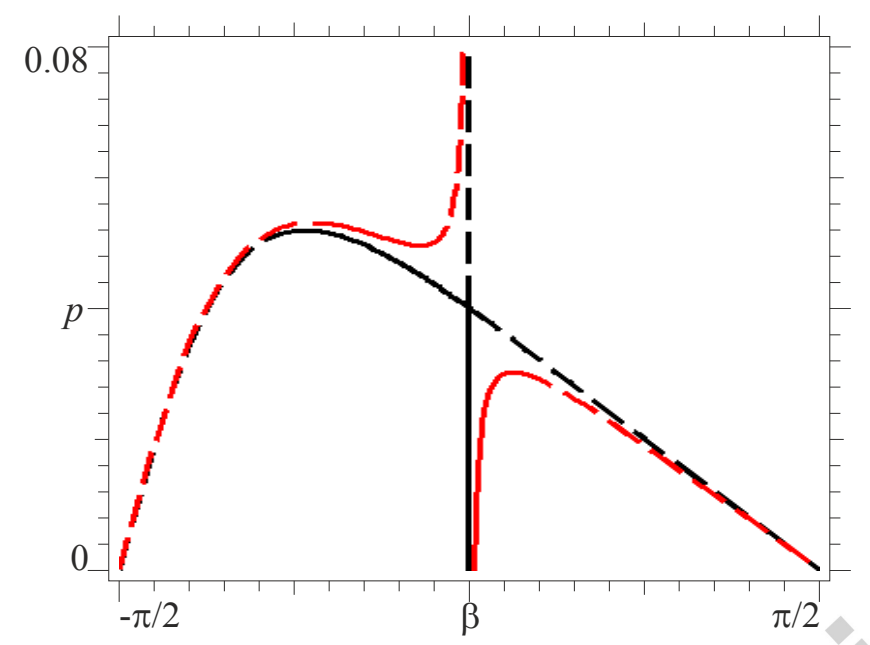

Fig. 5. (color online) Zoom of the bifurcation diagrams of both perfect and imperfect $(q=0.01)$ cases around the bifurcation point. $\alpha=0.8$, continuous $=$ stable, dashed $=$ unstable.

\section{THE EULER CRITICAL LOAD}

The Euler buckling load $p_{E}$ corresponds to the bifurcation point in the perfect case $q=0$. By noting that

$$
\left[1-\frac{1}{\sqrt{1+\alpha \sin (\beta)}}\right] \cos (\beta)=\frac{\alpha}{2} \beta-\frac{3 \alpha^{2}}{8} \beta^{2}+O\left(\beta^{3}\right), \quad \sin (\beta)=\beta+O\left(\beta^{3}\right)
$$

we obtain that the equilibrium equation in the perfect case becomes

$$
p \beta \cong \frac{\alpha}{2} \beta-\frac{3 \alpha^{2}}{8} \beta^{2} \Rightarrow\left\{\begin{array}{l}
\beta=0 \\
p \cong \frac{\alpha}{2}-\frac{3 \alpha^{2}}{8} \beta
\end{array}\right.
$$

so that

$$
p_{E}=\frac{\alpha}{2} \text {. }
$$

Note that $p_{E}=0.4$ for $\alpha=0.8$, a value that will be used in the following for reference.

By defining $\varepsilon_{E}=p_{E-} p$ we then obtain from (8)-(9) that approaching the bifurcation point the unstable saddle on the right of the rest position has the asymptotic expression

$$
\beta_{s} \cong \frac{8}{3 \alpha^{2}} \varepsilon_{E}
$$

i.e., it linearly approaches the rest position, as can be seen in Fig. 5. 


\section{THE KOITER CRITICAL LOAD}

The Koiter buckling load $p_{K}$ corresponds to the maximum point of the right branch of equilibrium points (see Figs. 4 and 5). To approximate it for small values of $q$ (and thus for small values of $\beta$, see Fig. 5) we note that

$$
-q \cos (\beta)=-q\left(1-\frac{\beta^{2}}{2}\right)+O\left(\beta^{3}\right)
$$

so that the equilibrium equation now provides

$$
p \cong \frac{\alpha}{2}-\frac{q}{\beta}+\left(\frac{q}{2}-\frac{3 \alpha^{2}}{8}\right) \beta .
$$

The maximum point can be obtained by solving $d p / d \beta=0$, which gives

$$
\beta_{K}=\frac{2}{3} \frac{\sqrt{6}}{\alpha} \sqrt{q}+O(q)
$$

and $(\alpha=0.8$ in the last expression)

$$
\begin{gathered}
p_{K}=p\left(\beta_{K}\right)=\frac{\alpha}{2}-\alpha \frac{\sqrt{6}}{2} \sqrt{q}+O(q), \quad \text { or } \\
p_{E}-p_{K} \cong \alpha \frac{\sqrt{6}}{2} \sqrt{q}=0.98 \sqrt{q} .
\end{gathered}
$$

Note that the difference between the Euler and the Koiter critical loads depends on the square root of the imperfection, a fact that is well known in the literature (see for example [18, pag. 21]) and which is at the base of the sensitive dependence of the critical load on the imperfections.

For $\alpha=0.8$ and $q=0.01$ we obtain $p_{K}=0.302$. The Koiter critical load in this case is $75 \%$ of the Euler prediction. The previous estimation of $p_{K}$ is very accurate, since by numerically solving $d p / d \beta=0$ without approximations we get $p_{K}=0.3014$.

By defining $\varepsilon_{K}=p_{K^{-}} p$ we obtain from (12)-(14) that approaching the bifurcation point the saddle has the asymptotic expression

$$
\beta_{s} \cong \beta_{K}+6^{1 / 4} \frac{4}{3} \frac{q^{1 / 4}}{\alpha^{3 / 2}} \sqrt{\varepsilon_{K}}
$$

i.e., it approaches the bifurcation point as the square root of the load, as can be seen in Fig. 5. The different asymptotic behaviour of the saddle in the perfect (Euler, eq. (10)) and imperfect (Koiter, eq. (15)) case explains the 'singular' behaviour of the perfect case, and the associated sensitivity to 
imperfections. Mathematically, this is a consequence of the fact that the point $(p, q)=\left(p_{E}, 0\right)$ is a singular point, in which the limit depends on the direction we approach it.

The asymptotic development method used in this and in the previous section has been largely used in the past in the 'Koiter analysis' of various structures [9, 19], thus having an 'historical' link with the Koiter theory.

\section{GLOBAL SAFETY, OR THE THOMPSON CRITICAL LOAD}

From (4) we get, in the conservative case,

$$
\dot{\beta}= \pm \sqrt{2[E-V(\beta)]}= \pm \sqrt{2\left\{E-\frac{[1-\sqrt{1+\alpha \sin (\beta)}]^{2}}{\alpha}+p[1-\cos (\beta)]+q \sin (\beta)\right\}}
$$

an expression which permits to draw the phase portrait without actually solving the equation of motion. An example is reported in Fig. 6a, together with the associated potential $V(\beta)$ (Fig. 6b).

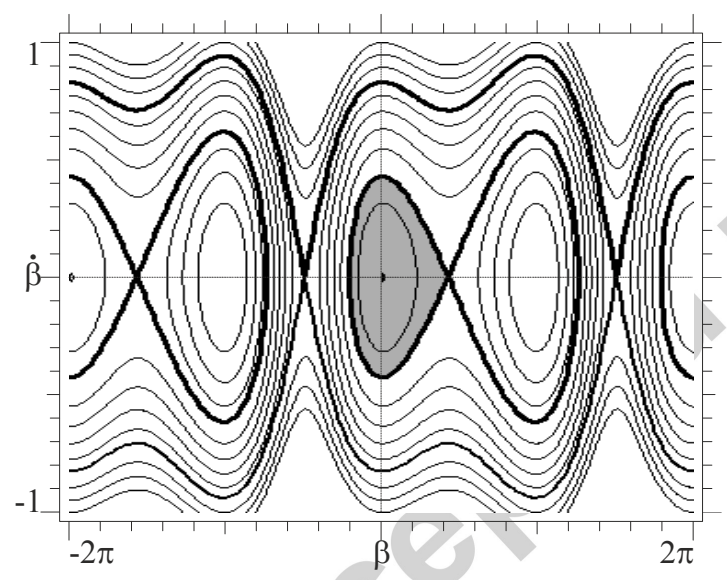

(a)

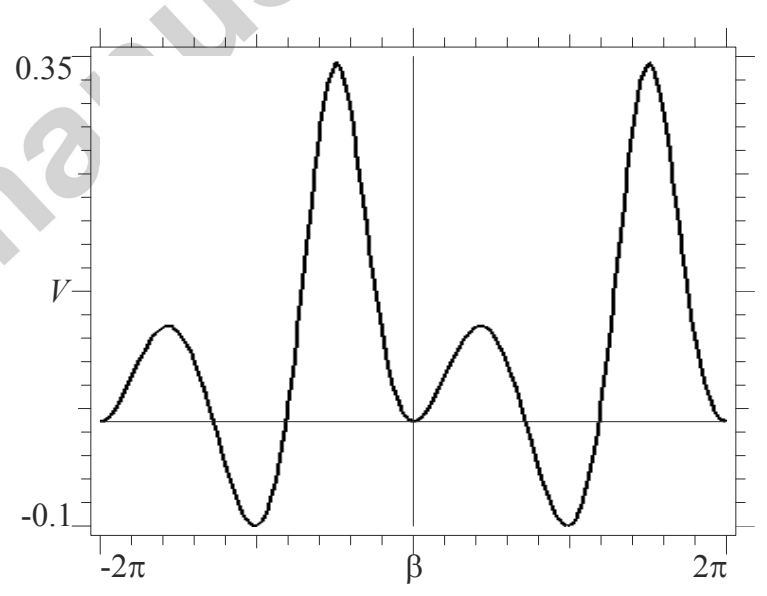

(b)

Fig. 6. (a) The phase portrait and (b) the potential $V(\beta)$ for $p=0.05, q=0.01$ and $\alpha=0.8$. Thick lines in (a) are homoclinic and heteroclinic orbits.

There are five types of solution, which are ordered for increasing values of the energy:

1) in-well periodic orbits, both in the well around $\beta=0$ (left well), which is the one of interest for the present analysis, and in the well around $\beta=\pi$ (right well);

2) two orbits homoclinic to the inner hilltop saddle, at $\beta_{s}=1.366$ in Fig. 6 , one on the right of the saddle and surrounding the right potential well, the other on the left and surrounding the left potential well (this will be of interest in the following);

3) cross-well periodic oscillations, turning around the two potential wells; 
4) two heteroclinic orbits of the outer hilltop saddle at $\beta=4.753$ in Fig. 6, one in the lower part of the phase space, implying anti-clockwise rotation, and the other in the upper part of the phase space, implying clockwise rotation;

5) clockwise and anti-clockwise rotations, encompassing all wells.

We are interested in the (left) potential well containing the equilibrium point close (in the perturbed case) to $\beta=0$, and in the associated homoclinic orbit.

The area within the left homoclinic orbit, which is reported in grey in Fig. 6a, is the safe region of the equilibrium position, which will become its "basin of attraction" if adding an infinitesimal damping. Borrowing concepts and tools from the dynamical integrity environment $[20,21]$, the magnitude of this region represents a measure of the global safety of the system, and provides information about its robustness with respect to dynamical imperfections, i.e., with respect to finite variations of initial conditions. It is clear that the larger is that area, the safer is the equilibrium position with respects to disturbances.

The main underlying idea of the global viewpoint (Thompson) ensues from the observation that the area of the safe basin shrinks as the axial load approaches the critical load, as clearly shown in the example reported in Fig. 7. Note that this property is true even in absence of perturbations (in fact, Fig. 7 corresponds to $q=0$ ), and thus, in some sense, the Thompson reasoning is independent of, and somehow bypasses, the Koiter approach (but this does not mean that the Koiter contribution was unimportant, of course!).

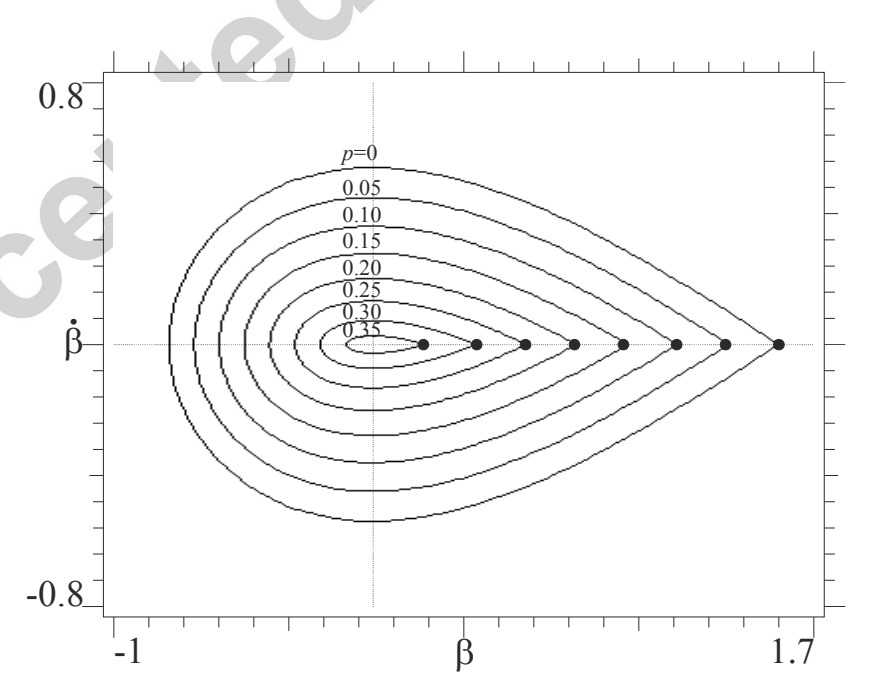

Fig. 7. The homoclinic loops for $q=0, \alpha=0.8$ and for different values of $p$. Note that the critical load is $p_{E}=0.4$. The dots are the saddle points $\beta_{s}$ for each loop.

Figure 7 clearly shows that, e.g., for $p=0.35$ the area is so small that the solution is unsafe for practical applications, as in the real word there are always finite, although small, disturbances. 
Note that the area is very small, but it is not null, in agreement with the fact that the solution is stable from a mathematical point of view. This observation clarifies the difference between the classical stability viewpoint (infinitesimal changes in initial conditions $\rightarrow$ the safe region is assumed to be infinitesimal) and the Thompson viewpoint (small but finite changes in initial conditions $\rightarrow$ the safe region must have a finite - though possibly small - extent).

Also note that, within a reliable global safety perspective, in the presence of actual dynamic excitations the occurrence of a finite safe region around the equilibrium point (i.e. the finite magnitude of the area of the safe basin) is not enough to guarantee the real dynamical integrity of the system, as it will be clarified in Part II of this work [17]. However, it is a sufficient concept for the goal pursued herein.

Since the area $A$ of the safe basin, and thus the global safety and practical reliability of the system, decrease as the axial load increases, to have an idea of the load carrying capacity of the system it is useful to draw the function $A(p)$.

To compute the area inside the homoclinic loop we note that on the homoclinic orbit the total energy $E$ (see eq. (4)) is that of the saddle $\beta_{s}$ (asymptotic expressions for $\beta_{s}$ are given in (10) for $q=0$ and in $(15)$ for $q \neq 0)$. But since the saddle is an equilibrium point, its kinetic energy vanishes and we have $E=V\left(\beta_{s}\right)$. Thus, from (16) we get that on the homoclinic orbit

$$
\dot{\beta}_{h}(\beta)= \pm \sqrt{2\left[V\left(\beta_{s}\right)-V(\beta)\right]} .
$$

Now let $\beta_{o}$ be the highest solution below $\beta_{s}$ of the transcendental equation $V\left(\beta_{s}\right)=V(\beta)$. $\beta_{o}$ is the lowest point reached by the homoclinic orbit, and thus the area inside the homoclinic loop is, by symmetry with respect to the $\dot{\beta}=0$ axis,

$$
A(p)=2 \sqrt{2} \int_{\beta_{o}}^{\beta_{s}} \sqrt{\left[V\left(\beta_{s}\right)-V(\beta)\right]} d \beta .
$$

This function is plotted in Fig. 8, where in the vertical axis we have reported the normalized area, i.e., $A(p) / A(0)(A(0)=1.720792$ for $\alpha=0.8)$. In the Thompson's dynamical integrity perspective, it represents a Global Integrity Measure $(G I M)[20,21]$ profile providing a (dimensionless) measure of the percentage reduction (with respect to the reference unloaded case) of the magnitude of the safe region with increasing load. Herein, it plays the simpler role of a measure of robustness of the stable equilibrium position under finite size perturbations.

Figure 8 much better than Fig. 7 shows that in the neighbourhood of the critical load $p_{E}=0.4$ the safe region is merely residual, and thus actually unsafe. The practical (Thompson) load carrying capacity is much less than $p_{E}=0.4$. For example, if we assume that $10 \%$ of the initial area is still 
acceptable (and this is of course a very low value in practice), then we have that the Thompson critical load is $p_{T}=0.238$ (see Fig. 8), i.e. $59 \%$ of the Euler prediction.

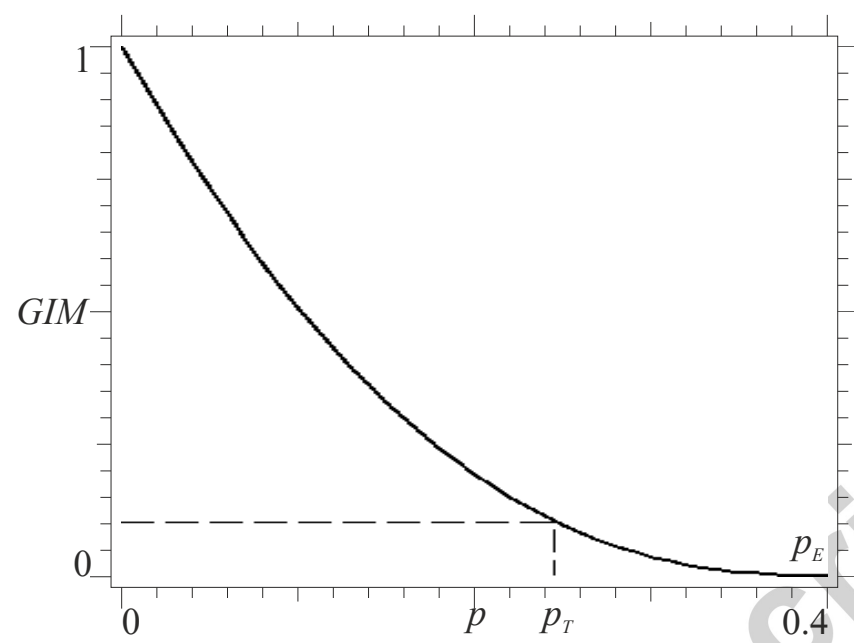

Fig. 8. The normalized area inside the homoclinic loops of Fig. 7 as a function of $p . q=0$ and $\alpha=0.8$. Note that the critical load is $p_{E}=0.4$, and that $\operatorname{GIM}(0.238)=0.1$.

The general picture delineated above for the perfect case $q=0$ does not change so much in the imperfect case, as shown in Fig. 9. We note that curves for $q \neq 0$ shift toward lower GIM values of a quantity which is roughly independent of $p$, and is instead proportional to $q$. The latter circumstance highlights how the static imperfection parameter $q$ (Koiter) already reduces the safe region extent, and thus the robustness of stable equilibria. This obviously entails that, for a given vertical load $p$, a dynamical imperfection (Thompson) lower than the one needed in the perfect case is sufficient to globally break the system safety.

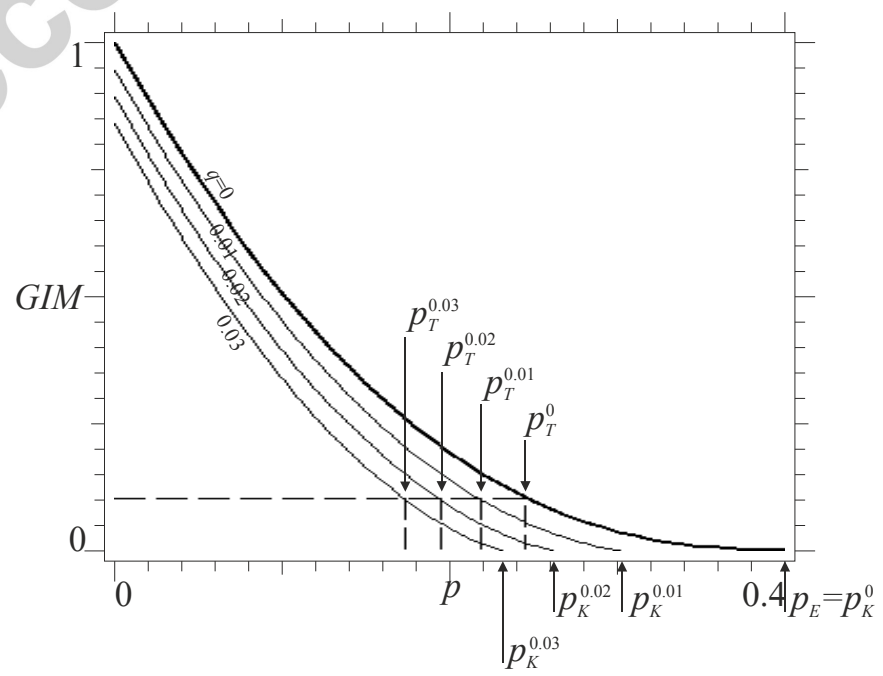

Fig. 9. The normalized area inside the homoclinic loops for $\alpha=0.8$ and for different values of $q$. The horizontal line at $G I M=10 \%$ schematically show how to compute the corresponding Thompson critical loads. 
The Koiter and Thompson critical loads are reported in Tab. 1, from which it is possible to appreciate even quantitatively the reduction of the load carrying capacity, with respect to the Euler one of the perfect system, due to Koiter perturbation, which has the historical primacy, and, independently, to Thompson one.

\begin{tabular}{|c|c|c|c|c|}
\hline \multirow{2}{*}{$q$} & \multirow{3}{*}{ Koiter load } & \multicolumn{3}{|c|}{ Thompson load } \\
\cline { 3 - 5 } & GIM=5\% & GIM=10\% & GIM=20\% \\
\hline 0.00 & 0.4 & 0.277 & 0.238 & 0.184 \\
& $(100 \%)$ & $(69 \%)$ & $(59 \%)$ & $(46 \%)$ \\
\hline 0.01 & 0.301 & 0.242 & 0.210 & 0.163 \\
& $(75 \%)$ & $(61 \%)$ & $(52 \%)$ & $(41 \%)$ \\
\hline 0.02 & 0.261 & 0.215 & 0.187 & 0.145 \\
& $(65 \%)$ & $(54 \%)$ & $(47 \%)$ & $(36 \%)$ \\
\hline 0.03 & 0.230 & 0.192 & 0.167 & 0.128 \\
& $(57 \%)$ & $(48 \%)$ & $(42 \%)$ & $(32 \%)$ \\
\hline
\end{tabular}

Tab. 1. Different critical loads (in parentheses the percentage with respect to the Euler load $p_{E}$, reported in the gray cell). The column $G I M=10 \%$ is illustrated in Fig. 9 .

The values in Tab. 1 show that the dynamical imperfections have a really major effect towards decreasing the critical load in the absence of static imperfection $(q=0)$, whereas their consequences are comparatively and progressively less important as the static imperfection increases. However, in such cases, they still produce meaningful additional reductions of the critical load with respect to that already entailed by the sole static imperfection.

Note that, while the Koiter critical load can be quantitatively determined upon fixing the value of the static imperfection $q$, the Thompson critical load can be determined only upon choosing the admissible residual safe region, i.e. after fixing the acceptable GIM, as clearly illustrated in Tab. 1. This corresponds just to fix the maximum allowed dynamical imperfections (change in initial conditions) which can be safely supported by the system. In this respect, both Koiter and Thompson theories share the property of being practically determinable with the exact knowledge (or an estimation) of the static $(q)$ and dynamical imperfection (GIM), respectively, an issue which is not easily achieved in practice, where the magnitude of imperfections is usually unknown and, moreover, has a large statistical dispersion.

Remark. It is interesting to see what happens to the GIM function and to the associated Thompson critical load by a nonlinear change of the system generalized coordinate.

To investigate this point, in addition to the variable $\beta$ used in the paper, which has the mechanical meaning of rotation angle (Fig. 1), we consider also $\delta=\sin (\beta)$, which has the mechanical meaning of lateral displacement of the top of the $\operatorname{rod}$, and $\chi$, defined by $\beta=\chi+\chi^{3}$, which has no mechanical 
meaning, but is one of the easiest nonlinear changes of variable. It has also the linear part otherwise it is singular for $\beta=\chi=0$. The three corresponding $\operatorname{GIM}(p)$ curves for $\alpha=0.8$ and $q=0$ are reported in Fig. 10 .

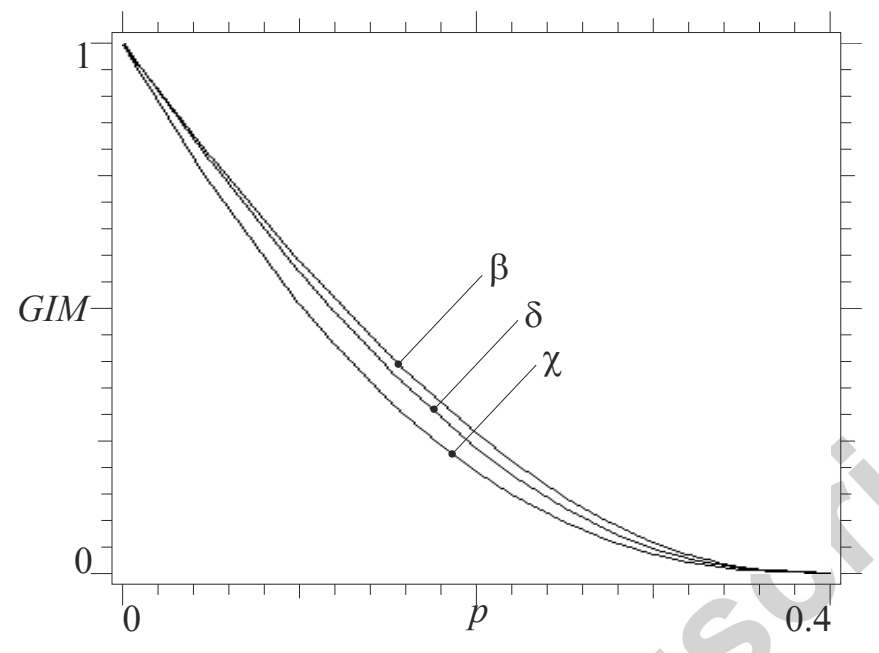

Fig. 10. The curves $\operatorname{GIM}(p)$ for $\alpha=0.8$ and $q=0$, for different system generalized coordinates.

Figure 10 shows that the three curves are distinct, and this confirms that from a mathematical point of view the robustness profile (i.e. the GIM as a function of the driving parameter) depends on the coordinate system, although all of them seem to have the same asymptotic behavior for $p \rightarrow p_{c r}$. However they are close, and from a mechanical point of view the differences are not so marked. Of course, for particular - let's say "special" - changes of coordinate the differences may be larger. So, our conclusion is that from a practical point of view, and certainly as far as we stay close to coordinate systems having a mechanical meaning, the GIM is almost independent of it. This means that the Thompson critical threshold is substantially independent, too.

The problem might arise if considering a "strange" change of coordinates possibly giving rise to very different curves. In such a case, one has any way to consider that the critical level on GIM (the one which provides the Thompson critical load) cannot be defined without referring to the mechanical nature of the perturbations themselves. In fact, for example, engineers may require that "the structure should support an accidental impulse equal to ....". This impulse is a quantity with physical dimensions, which may correspond to different abstract quantities assumed as generalized coordinates. However, when changing the coordinate systems, the threshold of admissibility must be changed accordingly in order for the mechanical Thompson threshold, i.e. the safety threshold against changes in physical initial conditions, to remain fixed. So, a unique information is obtained for the mechanical Thompson critical threshold while describing it through possibly different mathematical models. 


\subsection{Asymptotic developments of the safe region}

As Figs. 8 and 9 show, the most important part of the $\operatorname{GIM}(p)$ curves is the final one, where they approach the Euler or Koiter critical loads. In fact, it is just this part which determines how much the Thompson critical load is lesser than the bifurcation point (Euler or Koiter), and it is just the rate of convergence which determines the sensitivity to dynamic perturbations. Thus, it is interesting to study this part carefully.

The ending behaviour of the curves $\operatorname{GIM}(p)$ can be detected analytically by an asymptotic analysis. As smallness parameters we assume, as in Sects. 3 and $4, \varepsilon_{E}=p_{E}-p=\alpha / 2-p$ in the perfect (Euler) case $q=0$, and $\varepsilon_{K}=p_{K}-p$ in the imperfect (Koiter) case $q \neq 0$. Thus, both these parameters are positive and measure the closeness to the bifurcation point, i.e. the closeness of $p$ to the end of the curves $\operatorname{GIM}(p)$.

To obtain the asymptotic expressions of the area we start by developing $V(\beta)$ up to the third order in $\beta$ around the hilltop saddle $\beta_{s}$. This is motivated by the fact that close to the bifurcation point the homoclinic loop occurs in a close neighbourhood of $\beta_{s}$, as shown in Fig. 7. We have

$$
V(\beta) \cong V\left(\beta_{s}\right)+V^{\prime}\left(\beta_{s}\right)\left(\beta-\beta_{s}\right)+\frac{V^{\prime \prime}\left(\beta_{s}\right)}{2}\left(\beta-\beta_{s}\right)^{2}+\frac{V^{\prime \prime \prime}\left(\beta_{s}\right)}{6}\left(\beta-\beta_{s}\right)^{3} .
$$

Since $\beta_{s}$ is a hilltop saddle, we have $V^{\prime}\left(\beta_{s}\right)=0$ and $V^{\prime \prime}\left(\beta_{s}\right)<0$. Furthermore, in the neighbourhood of $\beta=0$ we have $V^{\prime \prime \prime}\left(\beta_{s}\right)=3 \alpha^{2} / 4$ up to infinitesimal $\varepsilon$ - and $q$-terms; this leading order value is used in the following.

Expression (18) becomes

$$
\begin{aligned}
A(p) & =2 \int_{\beta_{o}}^{\beta_{s}}\left(\beta_{s}-\beta\right) \sqrt{\left[-V^{\prime \prime}\left(\beta_{s}\right)-\frac{V^{\prime \prime \prime}\left(\beta_{s}\right)}{3}\left(\beta-\beta_{s}\right)\right]} d \beta= \\
& =2 \int_{\beta_{o}}^{\beta_{s}}\left(\beta_{s}-\beta\right) \sqrt{\left[-V^{\prime \prime}\left(\beta_{s}\right)-\frac{\alpha^{2}}{4}\left(\beta-\beta_{s}\right)\right]} d \beta .
\end{aligned}
$$

To the same order of approximation we have that $\beta_{o}$ is given by (it is just the solution of the radicand of (20) equated to zero)

$$
\beta_{o}=\beta_{s}-\frac{3 V^{\prime \prime}\left(\beta_{s}\right)}{V^{\prime \prime \prime}\left(\beta_{s}\right)}=\beta_{s}-\frac{4 V^{\prime \prime}\left(\beta_{s}\right)}{\alpha^{2}} .
$$

With this expression the integral in (20) can be computed in closed form, and the result is

$$
A(p)=\frac{24}{5} \frac{\left(-V^{\prime \prime}\left(\beta_{s}\right)\right)^{5 / 2}}{\left(V^{\prime \prime \prime}\left(\beta_{s}\right)\right)^{2}}=\frac{128}{15} \frac{\left(-V^{\prime \prime}\left(\beta_{s}\right)\right)^{5 / 2}}{\alpha^{4}} .
$$


In the perfect (Euler) case $q=0$ we have the asymptotic expression (10) for $\beta_{s}$. By inserting this expression in (22) and by developing the result in $\varepsilon_{E}$ series we have that the asymptotic expansion of the area $A(p)$ of the safe region around $p_{E}$ is ( $\alpha=0.8$ in the second expression)

$$
A(p) \cong \frac{128}{15} \frac{\varepsilon_{E}^{5 / 2}}{\alpha^{4}}=20.83 \varepsilon_{E}^{5 / 2}
$$

This expression shows how fast GIM goes to zero when $p \rightarrow p_{E}$ (remember that $\varepsilon_{E}=p_{E}-p$ ). It is worthy to note that the asymptotic exponent $5 / 2$ is more severe than the asymptotic exponent $1 / 2$ of the Koiter imperfections (see eq. (14)), showing how in the present case the system is more sensitive to global safety requirement than to the static Koiter perturbations, and confirming that the Thompson critical load is always lower than the Koiter critical load.

In turn, in the imperfect (Koiter) case $q \neq 0$ we have the asymptotic expression (15) for $\beta_{s}$. By inserting this expression in (22), remembering that $p_{K}$ has the asymptotic expansion (14), and by developing the result in $\varepsilon_{K}$ and $q$ series we have that the asymptotic expansion of the area $A(p)$ of the safe region around $p_{K}$ is ( $\alpha=0.8$ in the second expression)

$$
A(p) \cong \frac{128}{15} 6^{5 / 8} \frac{q^{5 / 8}}{\alpha^{11 / 4}} \varepsilon_{K}^{5 / 4}=48.30 q^{5 / 8} \varepsilon_{K}^{5 / 4}
$$

an expression which shows how fast GIM goes to zero when $p \rightarrow p_{k}$ (remember that $\varepsilon_{K}=p_{K}-p$ ). Note that now the power is $5 / 4$ instead of $5 / 2$, namely, the reduction of critical load corresponding to a given residual safe region is lower for the imperfect system (Koiter) than for the perfect one (Euler) - as confirmed by the numerical results in Tab. 1 -, the former being thus less sensitive than the latter to dynamical imperfections. This difference, which can be appreciated in Fig. 9, ensues from the different asymptotic behaviour of the saddles, which can be seen by comparing (10) with (15).

The effectiveness of asymptotic expressions (23) and (24) is shown in Fig. 11, where the real expressions (coming from Fig. 9) and the asymptotic ones are compared.

Finally, to further underline the differences between the three critical loads, and their dependence on the (small) magnitude of imperfections, we summarize the previously obtained asymptotic behaviours (eqs. (14), (23) and (24)):

$$
\begin{gathered}
p_{E}-p_{K} \cong c_{1} q^{1 / 2}, \\
p_{E}-p_{T} \cong c_{2} G I M_{c r}^{2 / 5}, \\
p_{K}-p_{T} \cong c_{3} \frac{G I M_{c r}^{4 / 5}}{q^{1 / 2}} .
\end{gathered}
$$

These expressions show that $p_{E}$ is a singular point with respect to both $q$ and $G I M_{c r}$, i.e., that $p_{E}$ is strongly sensitive to (static and dynamic) imperfections. Again, it is confirmed that the 
Thompson critical load is the most severe, being the lowest one $\left(c_{1}, c_{2}\right.$ and $c_{3}$ are positive constants).

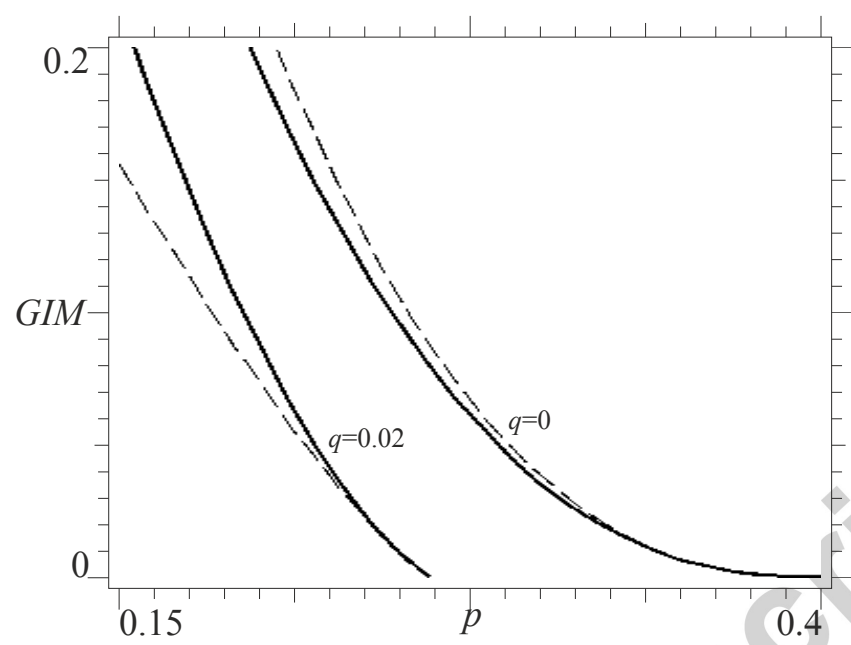

Fig. 11. The exact curves $\operatorname{GIM}(p)$ (thick) compared with their asymptotic approximations (thin and dashed) for $\alpha=0.8$ and for two different values of $q$.

\section{CONCLUSIONS}

The problem of load carrying capacity of axially loaded elastic structures has been reconsidered. Following an historical perspective corresponding to the advancements in the knowledge, three major contributions, due to outstanding researchers, have been identified.

1) Euler, who was the first to discover the loss of stability of equilibrium configurations. He considered perfect structures and obtained a bifurcation (buckling) of branching type (in the reference model of Fig. 1 it is a transcritical bifurcation due to the asymmetry of the system, but in the original Euler problem the bifurcation was a pitchfork due to the symmetry).

2) Koiter, who was the first to discover that the load carrying capacity is sensitive to static perturbations or imperfections, and that the actual bifurcation is of jumping type (saddle-node); in some sense he was a precursor - in the mechanical community - of the unfolding concept of the bifurcation theory, as well as of the structural stability issue.

3) Thompson, who was the first to highlight the importance of dynamic perturbations, which can be adequately described by the novel concept of dynamical integrity. He understood that stability is not enough for practical applications, since the perturbations of initial conditions are always of finite, although small, magnitude in the reality, and not infinitesimal as in the mathematical definition. Consequently, he understood that a local analysis is no longer sufficient, and that a global analysis is needed, with more complicated theoretical and practical treatments. 
By means of an archetypal unsymmetrical mechanical system, the principal aspects involved in the three estimations of the critical load have been highlighted in detail. The simplicity of the model permitted an analytical approach without the use of numerical simulations, even for the global analysis requested in the Thompson approach, a fact that allows a deep understanding of the phenomena occurring in the dynamical perspective.

Similarities and differences between the Koiter and Thompson 'corrections' to the Euler analysis have been highlighted.

By means of an asymptotic analysis it has been shown the sensitivity of the unperturbed (Euler) problem to the static (Koiter) and dynamic (Thompson) perturbations, as well as the sensitivity of the statically perturbed (Koiter) problem to dynamic perturbations. This is at the base of the fact that the Koiter critical load is significantly lower than the Euler critical load, and that the Thompson critical load is significantly lower than both Euler and Koiter critical loads.

Exploiting concepts and tools obtained in terms of system integrity within the nonlinear dynamics community, the basic concept of global safety of axially loaded systems has been discussed, along with its measure and its difference with respect to the classical stability concept, showing how fast the safe region shrinks to zero as the load approaches the critical threshold. In fact, only the basic aspect of the dynamical integrity perspective, which consists of allowing for finite size perturbations, has been used in this paper, since it is sufficient for highlighting the actual robustness of stable equilibria. As it will be shown in Part II of this work [17], the issue of system load carrying capacity and global safety in the presence of actual dynamic excitations requires full consideration of its highly variable dynamic response, with the ensuing effects in terms of reduction of dynamical integrity due to the erosion phenomena entailed by the possible occurrence of fractal basins of attraction.

However, as far as the problem of practical stability of structures is concerned, it is the authors' opinion that upon assuming the Thompson global viewpoint it can be considered as definitely understood from a theoretical point of view, although much work is still needed in the direction of practical applications of the underlying ideas.

Acknowledgement. The authors wish to thank one reviewer for very useful comments which permit to clarify an important point.

\section{REFERENCES}

[1] L. Euler, Methodus inveniendi lineas curvas maximi minimive proprietate gaudentes, sive solutio problematis isoperimetrici latissimo sensu accepti. Addentamentum 1: de curvis elasticis, Laussanae et Genevae, Apud Marcum-Michaelem, Bousquet et Socios, pp. 245-310, 1744.

[2] A.M. Lyapunov, The general problem of the stability of motion, Ph.D. Thesis, Moscow University, 1892. English translation: A.M. Lyapunov, 1992, The general problem of the stability of motion, London, Taylor \& Francis, 
1992.

[3] R.I. Leine, "The historical development of classical stability concepts: Lagrange, Poisson and Lyapunov stability," Nonlinear Dyn., vol. 59, pp. 173-182, 2010.

[4] W.T. Koiter, 1945, Over de stabiliteit van het elastisch evenwicht, Ph.D. Thesis, Delft University, 1945. English translation: W.T. Koiter, On the Stability of Elastic Equilibrium, NASA Technical Translation F-10, 833, Clearinghouse, US Dept. of Commerce/Nat. Bur. of Standards N67-25033, 1967.

[5] H.A. Mang, X. Jia, G. Hoenger, "Hilltop buckling as the A and $\Omega$ in sensitivity analysis of the initial postbuckling behavior of elastic structures," J. Civ. Eng. Manag., vol. 15, pp. 35-46, 2009.

[6] R. Thom, Structural Stability and Morphogenesis, W.A. Benjamin Inc., Massachusetts, 1972.

[7] E.C. Zeeman, Catastrophe Theory-Selected Papers 1972-1977. Reading, MA, Addison-Wesley, 1977.

[8] B. Budiansky, J.W. Hutchinson, "Dynamics buckling of imperfection-sensitive structures," Proc. of XI Inter. Congress Appl. Mech., Springer, Berlin, 636-651, 1964.

[9] M. Pignataro, N. Rizzi, A. Luongo, Bifurcation, Stability and Postcritical Behaviour of Elastic Structures, Elsevier Science Publishers, Amsterdam, 1990.

[10] W.T. Koiter, Elastic Stability of Solids and Structures, Edited by A. van der Heijden, Cambridge University Press, 2009.

[11] J.M.T. Thompson, Instability and Catastrophe in Science and Engineering, Wiley, 1982.

[12] M. Novak, “Aeroelastic galloping of prismatic bodies”, ASCE J. Eng. Mech., vol. 95, pp. 115-142, 1969.

[13] I. Elishakoff, "Elastic Stability: From Euler to Koiter There Was None Like Koiter", Meccanica, vol. 35, pp. 375$380,2000$.

[14] J.M.T. Thompson, "Chaotic phenomena triggering the escape from a potential well", Proceedings of the Royal Society of London A, vol. 421, pp. 195-225, 1989.

[15] M.S. Soliman, J.M.T. Thompson, "Integrity measures quantifying the erosion of smooth and fractal basins of attraction", J. Sound Vibr., vol. 135, pp. 453-475, 1989.

[16] A.N. Lansbury, J.M.T. Thompson, H.B. Stewart, "Basin erosion in the twin-well Duffing oscillator: Two distinct bifurcation scenarios," Int. J. Bif. Chaos, vol. 2, pp. 505-532, 1992.

[17] S. Lenci, G. Rega, "Load carrying capacity of systems within a global safety perspective. Part II: Attractor/basin integrity under dynamic excitations." submitted.

[18] J.M.T. Thompson, G.W. Hunt, A General Theory of Elastic Stability, John Wiley \& Sons, London, 1973.

[19] Sun Haihong, Chen Tieyun, "Koiter-boundary layer singular perturbation method for axial compressed stiffened cylindrical shells," Appl. Math. Mech., vol. 18, pp. 493-502, 1997.

[20] G. Rega, S. Lenci, "Identifying, evaluating, and controlling dynamical integrity measures in nonlinear mechanical oscillators,” Nonlin. Anal. T. M. \& A., vol. 63, pp. 902-914, 2005.

[21] G. Rega, S. Lenci, "Dynamical integrity and control of nonlinear mechanical oscillators," J. Vibr. Control, vol. 14, pp. 159-179, 2008. 
In this paper the dynamical integrity ideas have been applied to the problem of load carrying capacity of structures.

While the examples refer to an archetypal problem, the underlying ideas are very general

It is believed that they can applied in many applications, even far away from the engineering context. 